\title{
The Relationship Between Reimbursement and Quality of Care for Patients Hospitalized with Heart Failure
}

\author{
E.P. Havranek ${ }^{*}, 1,2,3$, P. Wolfe ${ }^{1}$, F.A. Masoudi ${ }^{1,2,3}$, J.M. Foody ${ }^{4}$, S.S. Rathore ${ }^{5,6}$ and H.M. Krumholz ${ }^{1,5}$ \\ ${ }^{I}$ Colorado Foundation for Medical Care, Denver, Colorado, USA \\ ${ }^{2}$ Division of Cardiology, Denver Health Medical Center, Denver, Colorado, USA \\ ${ }^{3}$ Division of Cardiology, University of Colorado Denver Health Sciences, Aurora, Colorado, USA \\ ${ }^{4}$ Division of Cardiovascular Medicine, Brigham and Women's Hospital, Harvard Medical School, Boston, Massachusetts, \\ USA \\ ${ }^{5}$ Section of Cardiovascular Medicine, Department of Internal Medicine, Yale University School of Medicine, New \\ Haven, Connecticut, USA
}

${ }^{6}$ MD/PhD Program, Yale University School of Medicine, New Haven, Connecticut, USA

\begin{abstract}
Data supporting the existence of a relationship between reimbursement and quality are limited. We assessed the association between quality of care for 34,318 patients hospitalized with heart failure across the US and heart failure Diagnosis-Related Group (DRG) payment for the 3,905 hospitals at which patients were admitted. Payment varied from $\$ 2606$ to $\$ 11,845$. We found a discontinuous relationship between documentation of ejection fraction and payment; below $\$ 4200$, there was a steep increase in rates of the quality indicator with increasing DRG payment (OR 1.15 for each $\$ 100$ increase, 95\% CI 1.12-1.18). For ACE inhibitor prescription, the increase in rates below the threshold was of borderline significance (OR 1.04 for each $\$ 100$ increase, 95\% CI 1.00-1.07). Hospitals with reimbursement below the threshold were more likely non-urban $(\mathrm{p}<0.001)$, public $(\mathrm{p}<0.0001)$, and without advanced cardiac facilities $(\mathrm{p}<0.0001)$, and had fewer full-time registered nurses per adjusted patient-day $(\mathrm{p}<0.0001)$. We conclude that hospitals with low rates of Medicare DRG-based reimbursement have lesser performance on a heart failure quality measure, perhaps because of difficulty investing in advanced cardiac facilities or maintaining patient care staffing.
\end{abstract}

\section{INTRODUCTION}

It is widely acknowledged that the U.S. healthcare system frequently fails to provide care based on the best available scientific evidence. In its landmark 2001 report Crossing the Quality Chasm [1], the Institute of Medicine placed responsibility for this failure squarely on the fundamental structure of the healthcare system, and called for system changes that would force improvements in quality of care. The report proposes that the structural elements of the healthcare system most in need of change are the incentives created by reimbursement, the legal and regulatory environment affecting quality, the training of the healthcare workforce, and the adoption of information technology. Since worker training and information technology infrastructure depend on availability of operating funds and capital, changes in the incentives created by reimbursement may have the greatest potential among these structural elements to effect improvements in quality.

Understanding the relationship between reimbursement and quality is therefore critical if we are to design systems that align financial incentives with desired outcomes of care. Although it has been assumed that higher levels of

*Address correspondence to this author at the Colorado Foundation for Medical Care, Denver, Colorado, USA; Tel/Fax: 1.303.436.7739;

E-mail: Edward.Havranek@dhha.org reimbursement are associated with higher quality of care, data supporting this relationship are limited. We therefore investigated the relationship between the reimbursement provided to a hospital for heart failure care under Medicare's Prospective Payment System and measures of the quality of care for Medicare heart failure patients. Medicare prospective payment to hospitals based on diagnosis-related groups (DRGs) represents a unique opportunity to study the effect of variation in reimbursement. The formula used to calculate reimbursement for individual hospitals, given in detail in the Appendix, contains a number of factors that cause reimbursement to vary widely from hospital to hospital. Although it is the intent of the Medicare system for this variation in reimbursement to account for local variation in hospitals' expenses, deviations are inevitable. We hypothesize that these deviations from fully accounting for hospital expenses result in improved profitability for some hospitals and diminished profitability for other hospitals, and that those with diminished profitability will be forced to make staffing and infrastructure decisions that adversely affect quality.

\section{MATERIALS AND METHODS}

Data Sources. Patient-level data for the study were obtained from the National Heart Failure project (NHF). The NHF was a quality initiative sponsored by the Centers for Medicare \& Medicaid Services (CMS) and was the heart failure component of the National Heart Care project; a separate component focused on acute myocardial infarction. As 
part of the initiative, data on the hospitalizations of 39,388 Medicare beneficiaries were obtained both from administrative records and from chart review. Charts were eligible for inclusion if patients were Medicare fee-for-service beneficiaries, the principal discharge diagnosis was heart failure as defined by International Classification of Diseases, Ninth Revision Clinical Modification [2] (ICD-9-CM) codes 428.x, $402.01,402.11,402.91,404.01,404.11$, and 404.9, and the date of discharge was between July 1, 2000 and June 30, 2001. Patients on chronic hemodialysis were excluded. After stratifying all eligible records from each state by age, race, gender, and hospital, 800 records per state were chosen at random. There were fewer than 800 discharges in Alaska, Hawaii, Idaho, Utah, Vermont, and Wyoming; for these states all charts were sampled. Charts were excluded if the patient was transferred to another medical facility or left against medical advice. We excluded 3122 patients under the age of 65 since these younger Medicare patients are not representative of elderly patients with heart failure. If a patient had a discharge selected more than once, one of the discharges was selected at random. Trained medical record reviewers abstracted clinical data from the charts and entered the information into an electronic database.

Hospital level data came from two sources. Structural information such as hospital size, staffing patterns, and teaching status was obtained for each patient's treating hospital through linkage with the American Hospital Association's 2000 Annual Survey of Hospitals. We classified hospital ownership into "for profit", "not for profit", or government. A hospital was classified as a teaching facility if it reported a medical school affiliation or reported membership in the Council of Teaching Hospitals. We used an index of Registered Nurse (RN) workload taken from the Annual Survey; this index is the total yearly number of RN hours per patient-day adjusted for the hospital's outpatient volume (adjusted patient days $=$ inpatient days + [inpatient days $\mathrm{x}$ (outpatient revenue/inpatient revenue)]). The hospital level data necessary to calculate Medicare payment to a hospital under the inpatient Prospective Payment System for an admission was obtained from publicly available CMS files for fiscal year 2000 [3]. The formula used to calculate an individual hospital's Diagnosis-Related Group (DRG) payment is presented in the Appendix.

Quality Measures. We measured quality with two measures of process of care. We chose to rely on process measures rather than outcome measures such as mortality for this hospital-level analysis because the number of outcomes per hospital is too small to allow meaningful inferences to be made, especially for the small hospitals that are of particular interest for this analysis. We chose two process measures that are widely accepted for defining quality of care for hospitalized patients with heart failure $[4,5]$, the proportion of patients for whom left ventricular function has been assessed and the proportion of patients with left ventricular systolic dysfunction who receive angiotensin converting enzyme (ACE) inhibitors. These measures are further defined in the Box 1.

Statistical Analysis. Because it is expected that performance on the quality indicators is clustered by hospital, we used a random effects hierarchical logistic regression models to identify predictors of ejection fraction documentation and
ACE inhibitor prescription. Results were calculated using probability weights (the inverse sampling fraction for each state) in order to obtain estimates for the quality indicators that are representative of all heart failure admissions nationwide during the sampling period. In order to determine the independent contribution of reimbursement level (analyzed categorically in increments of $\$ 100$ ), we adjusted for hospital level variables identified as potentially important from prior studies or potential confounders of DRG payment. These variables were teaching status, ownership (a proxy variable for hospitals receiving disproportionate share payment), presence of advanced cardiac services, presence of advanced cardiac services, and urban/rural location in the initial model. Because of the strength of the association between urban/rural location and DRG payment, the geographical variable was not included in the final model. We also included as an independent variable the percentage of the hospital's reimbursement dollars from Medicare, since level of DRG payment is likely to disproportionately affect hospitals whose revenue stream is more dependent on Medicare payments. Finally, we assessed the contribution of variables related to hospital staffing patterns as potential effect mediators.

\section{Box 1. Definitions of Quality Measures}

1. Documentation of assessment of left ventricular function: Proportion of patients with a principal discharge diagnosis of heart failure who had an assessment of left ventricular function documented in the medical record.

- Exclusion criteria: age $<65$, on chronic renal dialysis, discharged to another acute care facility, discharged against medical advice, died during the hospitalization, admitted on either an ACE inhibitor or an angiotensin receptor blocker, documented plan for left ventricular function assessment after discharge.

2. Appropriate use of ACE inhibitors:Proportion of patients with a principal discharge diagnosis of heart failure who had documented left ventricular systolic dysfunction (ejection fraction $<40 \%$ or a narrative statement consistent with an ejection fraction $<40 \%$ ) and were prescribed an ACE inhibitor at the time of discharge.

- Exclusion criteria: age < 65, on chronic renal dialysis, discharged to another acute care facility, discharged against medical advice, died during the hospitalization, documented plan for left ventricular function assessment after discharge, admitted on either an ACE inhibitor or an angiotensin receptor blocker, discharged on an angiotensin receptor blocker, enrolled in a clinical trial assessing alternative to an ACE inhibitor.

We did not include patient demographic or clinical factors as independent variables in the models. Adjustment for patient factors is appropriate when patients are at differential risk for an outcome. In the current analysis all patients were at equal "risk" for the "outcomes" of receiving an ejection fraction determination or ACE inhibitor prescription since by consensus all included patients should have received them; both the Joint Commission on Accreditation of Healthcare Organizations and the Centers for Medicare \& Medicaid Services have relied on these definitions for quality assessment in heart failure.

We plotted the empirical logit (the exponential term from the logistic regression) of the outcomes against DRG price, since this parameter gives linear relationships and is thus more easily interpreted graphically. These plots suggested the relationship to DRG price was not described by a single line. We used piecewise linear models to determine the loca- 
tion of the knot (point where the slope of the relationship changes). Because the location of the knot was data driven, it was slightly different for the two measures.

All analyses were conducted using MLwiN version 1.1 (Institute of Education, London, U.K.).

\section{RESULTS}

The charts of 39,388 patients were available for the NHF sample. After applying the general exclusion criteria then the inclusion and exclusion criteria for each measure, 15,208 patients were eligible for the ejection fraction documentation indicator and 4,047 patients were eligible for the ACE inhibitor prescription indicator. Exclusions are shown in Fig. (1).

The initial subset of 34,318 had an age distribution typical for Medicare patients (28.2\% 65-74 years, 42.8\% 75-84 years, $29.0 \%>85$ years) and was $58.5 \%$ female. The pa- tients were $85.2 \%$ Caucasian, $9.7 \%$ black, and $5.1 \%$ in other racial groups. $3.5 \%$ of patients were identified as Hispanic. Details of the baseline characteristics of the NHF population have been published previously [6].

The characteristics of the 3,905 acute care facilities at which the NHF patients were hospitalized are shown in Table 1. DRG payment for heart failure admissions varied widely, from $\$ 2606$ to $\$ 11,845$ per admission.

As described in the Methods section, the relationship between DRG reimbursement and performance on the quality measures was investigated graphically and it appeared to be best described by two lines with differing slope. Piecewise linear regression demonstrated that the difference in slopes of the two lines was indeed significant $(p<0.05)$; the location of the knot (point where the slope of the relationship changes) was determined to be at $\$ 4200$ for documentation of ejection fraction. The characteristics of the hospitals with

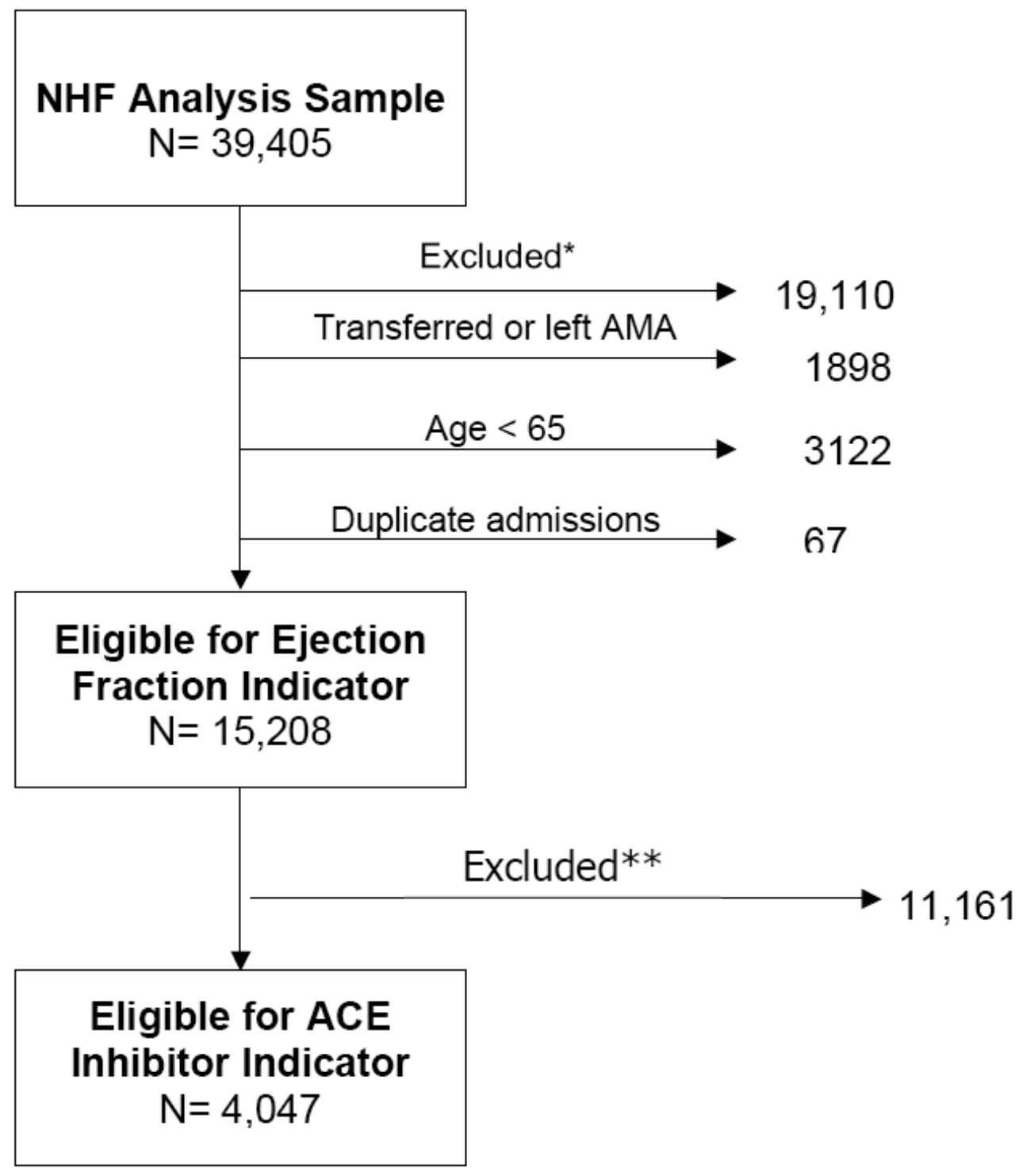

*Died, on ACE inhibitor prior to admission, plan for ejection fraction evaluation after discharge (see Box 1)

**No ejection fraction documented or systolic dysfunction not present, on angiotensin receptor blocker (see Box 1)

Fig. (1). Study Exclusions. 
DRG 127 reimbursement above and below the threshold level are compared in Table 2 . In general, hospitals below the threshold tended to be smaller, rural hospitals without advanced cardiac services and were more likely to be public facilities.

Table 1. Characteristics of Study Hospitals

\begin{tabular}{|c|c|}
\hline $\mathbf{N}$ & 3905 \\
\hline \multicolumn{2}{|l|}{ MSA* [N (\%)] } \\
\hline$<100,000$ & $40(1.1)$ \\
\hline $100,000-249,999$ & $311(8.3)$ \\
\hline $250,000-499,999$ & $335(8.9)$ \\
\hline $500,000-999,999$ & $325(8.7)$ \\
\hline $1,000,000-2,499,999$ & $571(15.2)$ \\
\hline$>2,500,000$ & $532(14.2)$ \\
\hline Non MSA & $1636(43.6)$ \\
\hline \multicolumn{2}{|l|}{ Teaching $[\mathrm{N}(\%)]$} \\
\hline Yes & $773(19.8)$ \\
\hline No & $3132(80.2)$ \\
\hline \multicolumn{2}{|l|}{ Ownership [N (\%)] } \\
\hline Government & $835(22.3)$ \\
\hline For profit & $526(14.0)$ \\
\hline Not for profit & $2389(63.7)$ \\
\hline \multicolumn{2}{|l|}{ Cardiac Facilities [N (\%)] } \\
\hline $\mathrm{OHS}^{\dagger}$ & $831(25.4)$ \\
\hline $\mathrm{CCL}^{\ddagger}$ & $639(19.5)$ \\
\hline Other & $1803(55.1)$ \\
\hline Medicare inpatient-days/ total inpatient-days & $0.51 \pm 0.14$ \\
\hline Beds (Mean \pm STD) & $188.6 \pm 178.6$ \\
\hline RN FTE/ adj patient-day** & $6.4 \pm 3.8$ \\
\hline
\end{tabular}

*Metropolitan Statistical Area. ${ }^{\dagger}$ Open heart surgery. ${ }^{\ddagger}$ Cardiac catheterization laboratory. **Registered nurse full-time equivalents per adjusted patient-day; see text for definition of adjusted patient-day.

For prescription of an ACE inhibitor, piecewise linear regression showed a borderline significant change $(\mathrm{p}=0.05)$ in slope for DRG reimbursement below a threshold of $\$ 4500$; for each increase of $\$ 100$ below the threshold, performance improved by approximately $4 \%$.

As described in the Methods, we investigated the association between reimbursement and teaching status, ownership, presence of advanced cardiac services, urban/rural location, the percentage of the hospital's reimbursement dollars from Medicare, and hospital staffing patterns; all these variables were significantly associated with level or reimbursement. All variables significant in the multivariable regression models are shown in Table 3. DRG reimbursement below the threshold of $\$ 4200$, level of registered nurse staffing, status as a teaching hospital, and the percentage of the hospital's inpatient days reimbursed by Medicare were all independent predictors of performance on the quality measure. For each increase of $\$ 100$ below the threshold, performance on the measure improved by approximately $15 \%$.
Table 2. Comparison of Hospitals Characteristics Above and Below DRG 127 Reimbursement Threshold

\begin{tabular}{|c|c|c|c|}
\hline & $<\$ 4200$ & $>\$ 4200$ & $\mathbf{P}$ \\
\hline MSA* $[\mathrm{N}(\%)]$ & & & $<0.0001$ \\
\hline$<100,000$ population & $19(1.1)$ & $21(1.0)$ & \\
\hline $100,000-250,000$ & $127(7.5)$ & $184(8.9)$ & \\
\hline $250,000-500,000$ & $109(6.5)$ & $226(11.0)$ & \\
\hline $500,000-1,000,000$ & $70(4.2)$ & $255(12.4)$ & \\
\hline $1,000,000-2,500,000$ & $53(3.1)$ & $518(25.1)$ & \\
\hline $2,500,00+$ & $26(1.5)$ & $506(25.1)$ & \\
\hline Non MSA & $1284(76.1)$ & $532(17.1)$ & \\
\hline Teaching [N (\%)] & & & $<0.0001$ \\
\hline Yes & $126(6.8)$ & $647(31.4)$ & \\
\hline No & 1717(93.2) & $1415(68.6)$ & \\
\hline Ownership [N (\%)] & & & $<0.0001$ \\
\hline Government & $529(31.3)$ & $306(14.8)$ & \\
\hline For profit & $187(11.1)$ & $339(16.4)$ & \\
\hline Not for profit & $972(57.6)$ & $1417(68.7)$ & \\
\hline Facilities [N (\%)] & & & $<0.0001$ \\
\hline $\mathrm{OHS}^{\dagger}$ & $124(8.2)$ & $707(40.0)$ & \\
\hline $\mathrm{CCL}^{\ddagger}$ & $199(13.2)$ & $440(24.9)$ & \\
\hline Other & $1181(78.5)$ & $622(35.2)$ & \\
\hline $\begin{array}{l}\text { Medicare inpatient-days/ } \\
\text { total inpatient-days }\end{array}$ & $0.55 \pm 0.13$ & $0.48 \pm 0.14$ & $<0.0001$ \\
\hline Beds (Mean \pm STD) & $110.3 \pm 102.6$ & $252.8 \pm 200.7$ & $<0.0001$ \\
\hline RN FTE/ adj patient-day** & $5.5 \pm 3.4$ & $7.2 \pm 3.9$ & $<0.0001$ \\
\hline
\end{tabular}

Table 3. Odds Ratios with $95 \%$ Confidence Intervals from Hierarchical Regression Model

\begin{tabular}{|c|c|c|}
\hline & $\begin{array}{c}\text { Ejection Fraction } \\
\text { Documented }\end{array}$ & $\begin{array}{c}\text { ACE Inhibitor } \\
\text { Prescribed }\end{array}$ \\
\hline \hline $\begin{array}{c}\text { Reimbursement below t } \\
\text { hreshold, per \$100* }\end{array}$ & $\begin{array}{c}1.15 \\
(1.12-1.18)\end{array}$ & $\begin{array}{c}1.04 \\
(1.00-1.07)\end{array}$ \\
\hline Teaching hospital & $\begin{array}{c}1.63 \\
(1.44-1.84)\end{array}$ & \\
\hline $\begin{array}{c}\text { RN FTE/adj } \\
\text { patient-day** }\end{array}$ & 1.06 & \\
\hline $\begin{array}{c}\text { Medicare inpatient-days/ } \\
\text { total inpatient days }\end{array}$ & $(1.04-1.08)$ & 1.65 \\
\hline
\end{tabular}

* The threshold was $\$ 4200$ for the "ejection fraction documented" quality measure and $\$ 4500$ for the "ACE inhibitor prescribed" quality measure.

**Registered nurse full-time equivalents per adjusted patient-day; see text for definition of adjusted patient-day.

Urban versus rural hospital location is a major determinant of several parameters in the DRG formula, raising the 
question of whether the relationship between reimbursement and quality is the result of confounding by rural/urban location. In order to determine if reimbursement level is associated with quality regardless of location, we estimated the model again separately in the urban and rural subsets. Results of this analysis are shown in Table 4.

Table 4. Odds Ratios from Hierarchical Regression Models for EF Measured Subset on Rural/Urban

\begin{tabular}{|c|c|c|}
\hline & Urban & Rural \\
\hline \hline $\begin{array}{c}\text { Reimbursement below } \\
\text { threshold, per } \$ 100^{*}\end{array}$ & $\begin{array}{c}1.07 \\
(1.03-1.11)\end{array}$ & $\begin{array}{c}1.17 \\
(1.11-1.22)\end{array}$ \\
\hline $\begin{array}{c}\text { Teaching } \\
\text { hospital }\end{array}$ & $\begin{array}{c}1.42 \\
(1.24-1.63)\end{array}$ & $\begin{array}{c}2.08 \\
(1.41-3.07)\end{array}$ \\
\hline $\begin{array}{c}\text { RN FTE/adj } \\
\text { patient-day** }\end{array}$ & 1.04 & 1.06 \\
$(1.01-1.06)$ & $(1.02-1.09)$ \\
\hline $\begin{array}{c}\text { Medicare inpatient-days/ } \\
\text { total inpatient days }\end{array}$ & 1.00 & 1.00 \\
$(1.00-1.00)$ & $(1.00-1.00)$ \\
\hline
\end{tabular}

Note: None of the predictors was significant in a similar model for ACEI, with the exception of For Profit $(\mathrm{OR}=0.65(0.50,0.86))$ in the Urban subset; this should be interpreted with caution.

For the ejection fraction measurement the slope of DRG reimbursement below the threshold remained significant in both subsets: OR=1.07 $(1.03,1.11)$ in the urban subset, $\mathrm{OR}=1.17(1.11,1.22)$ in the rural subset. For the ACE inhibitor prescription measure, the findings were also similar to those in the non-stratified model. None of the explanatory variables was significant except for "For Profit" in the urban subset: $\mathrm{OR}=0.65(0.50,0.86)$.

\section{DISCUSSION}

The principal finding of our study was that the rate at which a hospital is reimbursed under Medicare's Prospective Payment System for heart failure independently predicted the hospital's performance on two key indicators of quality of care for heart failure. The fact that the effect was significantly more pronounced for the ejection fraction documentation indicator in hospitals where Medicare represented a larger proportion of the hospital's revenue supports is further evidence for the existence of a relationship. There was an independent positive effect on this indicator of teaching hospital status above and beyond the effect of teaching hospital status on DRG reimbursement, and there was an independent positive effect on this indicator of having more registered nurse hours per adjusted patient day.

Our investigation of association between quality measures and other structural variables such as teaching status, nursing staffing, importance of Medicare reimbursement, and advanced cardiac facilities should be viewed as tentative, demonstrating that mediation of the relationship by presence of advanced cardiac facilities and by nursing staffing is plausible but by no means definitive. The interdependent relationships among these variables are complex.

Medicare reimbursements to hospitals changed in 1983 from a retrospective fee-for-service payment to a prospective payment system under which hospitals receive a flat amount for an admission, with the amount determined by the diagnosis responsible for the patient's hospitalization. These changes were introduced in an attempt to control costs by creating incentives to decrease length of stay and intensity of services.

Soon after its introduction, the effect of this Prospective Payment System on quality of care was a focus of detailed study $[7,8]$. For five index conditions, care improved following its introduction [9], continuing secular trends in quality improvement that had already begun [10]. In addition, risk-adjusted 30-day mortality declined significantly from $16.5 \%$ in $1981-1982$ to $15.4 \%$ in $1985-1986$ [11]. Contemporary smaller scale studies tended to confirm these results $[12,13]$.

More recent studies have found contrasting results. Shen [14] studied the relationship between changes in mortality for acute myocardial infarction and the changes in reimbursement under the Prospective Payment System for 1985 compared with 1990 and 1990 compared with 1994. For the 1990-1994 but not the 1985-1990 comparison, there was a statistically significant increase in mortality associated with decrement in reimbursement. A possible explanation for the contrast between this study and those of the RAND investigators is the author's finding that there was a greater relative financial impact on hospitals from the Prospective Payment System after 1990. Cutler [15] used data from Medicare patients hospitalized with acute myocardial infarction between 1981 and 1988 and found in increase in in-hospital mortality of approximately 0.5 percentage points for each standard deviation change in payment. Both studies were hampered by an inability to adequately adjust for mortality risk.

Whether or not variation in DRG-based reimbursement is associated with variation in quality of care has not been investigated.

The literature suggests several possible explanations for the linkage between lower reimbursement and decrements in quality. First, greater financial pressure associated with inadequate reimbursement might distract hospitals from being able to focus on quality. Bradley and colleagues [16] performed in depth interviews at eight hospitals that had a broad range of quality performance and hospital characteristics. Using qualitative analysis techniques, they identified goal setting, administrative support, clinician support, performance initiatives, use of data, and contextual factors as predictors of quality of care. Among the contextual factors, organizational turbulence, which included turnover of key personnel and financial stress, was identified as restraining quality improvement.

Second, financial stress might affect staffing patterns that in turn might have an adverse effect on the ability to provide high quality care. Particular attention has been focused on nursing, with several studies finding lower rates of inhospital complications and mortality associated both with overall nursing availability and the quantity of care provided by registered nurses [17-20].

Finally, relatively inadequate reimbursement might limit the ability to invest in technology. McClellan and Cutler [21] investigated the relationship between measures of what they term "insurance generosity" and diffusion of the use of coronary angioplasty following myocardial infarction in Medicare patients. They found that hospitals were less likely to initiate angioplasty programs and angioplasty was less likely 
to be used in areas where hospital reimbursement rates were regulated and where health maintenance organization participation was high. Angioplasty was more likely to be performed on Medicare patients in areas with high rates of uninsured patients, presumably because hospitals increased volume of reimbursed procedures to compensate for unreimbursed procedures.

There are several limitations to our study. First, we looked at marginal revenue per heart failure admission and not total revenue for heart failure care. We do not see this as a significant factor because unlike with elective procedures, hospitals have limited ability to increase the number of heart failure admissions to compensate for smaller payment per admission.

Second, there is co-linearity between PPS-based reimbursement rate and other hospital factors that might have an effect on quality of care. As can be seen in the formula presented in the Appendix, prospective payment varies systematically with teaching hospital status, level of indigent care provided, and urban versus non-urban location. We controlled for teaching hospital status and found that both teaching hospital status and DRG-based payment predicted quality performance independently. Although no single hospital characteristic distinguishes hospitals providing a disproportionate share of indigent care, such hospitals tend to be public and not-for-profit. After controlling for ownership, payment still remained an independent predictor. Because of the high degree of correlation between DRG-based payment and urban versus non-urban location, we have been unable to fully separate geographic from reimbursement factors. We also did not address provider level factors affected by important structural factors that may have an effect on quality. Chief among these is provider specialty. Hospitals able to provide advanced cardiac facilities are likely to attract a greater number of specialists, who in turn might provide care of greater quality [22-24].

Finally, as with any retrospective analysis of observational data, unmeasured confounders are almost certainly present.

\section{CONCLUSIONS}

There is a discontinuous relationship between reimbursement rates for heart failure inpatient care and indicators of quality of care for heart failure, with a decline in quality below a threshold in the mid range of reimbursement rates and no improvement in quality above the threshold. In the case of heart failure, anecdotal evidence suggests that current reimbursement patterns may be detrimental to efforts to improve quality [25], because expenditures on quality improvements efforts are associated with declines in admission rates and therefore in revenue.

Our study is not an indictment of the Prospective Payment System. It does, however, raise the possibility that reimbursement under prospective payment might be restructured in such a way that overall quality of care for the system might be improved without a net increase in cost. In the case of heart failure, a policy restructuring likely to fit these needs is that of "bundled payments" [26], under which hospitals and providers are jointly reimbursed for "episodes of care" that extend beyond hospital discharge. Clearly, extension of our results into other common medical conditions is warranted prior to considering changes in policy.

\section{ACKNOWLEDGEMENTS}

The analyses upon which this publication is based were performed under Contract Number 500-99-C001 entitled "Utilization and Quality Control Peer Review Organization for the State of Colorado" sponsored by the Centers for Medicare \& Medicaid Services (CMS), formerly known as the Health Care Financing Administration, Department of Health and Human Services. The content of the publication does not necessarily reflect the views or policies of the Department of Health and Human Services, nor does mention of trade names, commercial products, or organizations imply endorsement by the U.S. government. The authors assume full responsibility for the accuracy and completeness of the ideas presented. This article is a direct result of the Health Care Quality Improvement Program initiated by CMS, which has encouraged identification of quality improvement projects from analysis of patterns of care, and therefore required no special funding on the part of this contractor. Ideas and contributions to the author concerning experiences in engaging with issues presented are welcomed.

\section{APPENDIX}

Medicare payment to a hospital under the Inpatient Prospective Payment System for an admission is calculated as follows":

$\underline{D R G}$ payment $^{* *}=[\underline{D R G \text { weight }}] x[1+\underline{I M E}+\underline{D S H}] x$ [capital payment + operating payment]

DRG weight $=$ Diagnosis-Related Group (DRG) weight. This factor is set for each DRG and is based on the average resources used to treat Medicare patients with a diagnosis within that group.

$\underline{I M E}=$ Indirect Medical Education adjustment. This factor applies only to teaching hospitals and is established for the hospital based on the ratio of residents to hospital beds. This factor currently provides a $5.5 \%$ increase in the IME payment for every $10 \%$ increase in the resident-to-bed ratio.

$\underline{D S H}=$ Disproportionate SHare adjustment. This factor applies to hospitals providing a significant volume of care to indigent patients and is based on the levels of Medicaid, Social Security disability Medicare, and state/local funding.

\section{Capital payment $=$ SCS $\times$ GAF $\times$ LUA $\times$ COLA}

$\underline{\mathrm{SCS}}=$ Standardized Capital Share. This is a single factor for the entire country and is set annually for the coming year.

$\underline{\mathrm{GAF}}=$ Geographic Adjustment Factor. These factors are set annually for the coming year. Factors for rural counties are set by state and factors for urban areas are set individually.

LUA $=$ Large Urban Add-on. This is a single factor for the entire country and is applied only to hospitals in large (population greater than one million) urban areas.

\footnotetext{
${ }^{*}$ See www.cms.hhs.gov/providers/hipps/default.aps

** In addition to this base DRG payment, individual cases may be eligible for extra payment if the case qualifies as an outlier
} 
COLA $=$ Cost Of Living Adjustment. This factor applies only to hospitals in Hawaii and Alaska.

$$
\text { Operating payment }=(\text { SLS } \times \text { WI })+(\text { SNLS } \times \text { COLA })
$$

$\underline{\text { SLS }}=$ Standardized Labor Share. Two levels of this factor are established for the country, one for urban areas and one for non-urban areas.

$\underline{\mathrm{WI}}=$ Wage Index. These factors are set annually for the coming year. Factors for rural counties are set by state and factors for urban areas are set individually, and are based on average hourly hospital wages in the area in question.

$\underline{\text { SNLS }}=$ Standardized Non-Labor Share. This is a single factor for the entire country and is set annually for the coming year.

\section{REFERENCES}

[1] Institute of Medicine Committee on Quality of Health Care in America. Crossing the quality chasm: A new health system for the 21st century. Washington, D.C: National Academy Press, 2001; 3960

[2] Hopkins CA, Hart AC. 2004 International Classification of Diseases 9th Revision Clinical Modification. 6th ed. Eden Prairie, MN: Ingenix, 2003.

[3] Huang Z. Calculating hospital specific DRG payments. Research Data Assistance Center. Minneapolis, MN 2003.

[4] Krumholz HM, Baker DW, Ashton CM, et al. Evaluating quality of care for patients with heart failure. Circulation 2000; 101: e122-40.

[5] Measuring and improving quality of care: A report from the American Heart Association/American College of Cardiology First Scientific Forum on Assessment of Healthcare Quality in Cardiovascular Disease and Stroke. Circulation 2000; 101: 1483-93.

[6] Havranek EP, Masoudi F, Westfall K, Wolf P, Ordin DL, Krumholz HM. The spectrum of heart failure in older patients: Results from the National Heart Failure project. Am Heart J 2002; 143: 412-7.

[7] Kahn K, Rubenstein L, Draper D, et al. The effects of the DRGbased prospective payment system on quality of care for hospitalized medicare payments. An introduction to the series. JAMA 1990; 264: 1953-5.

[8] Draper D, Kahn K, Reinisch E, et al. Studying the effects of the DRG-based prospective payment system on quality of care: Design, sampling, and fieldwork. JAMA 1990; 264: 1956-61.

[9] Kahn K, Rogers W, Rubenstein L, et al. Measuring quality of care with explicit process criteria before and after implementation of the DRG-based prospective payment system. JAMA 1990; 264: 196973.
[10] Rogers W, Draper D, Kahn K, et al. Quality of care before and after implementation of the DRG-based prospective payment system. A summary of effects. JAMA 2004; 264: 1989-94.

[11] Kahn K, Keeler E, Sherwood M, et al. Comparing outcomes of care before and after implementation of the DRG-based prospective payment system. JAMA 1990; 264: 1984-8.

[12] Fitzgerald J, Moore P, Dittus R. The care of elderly patients with hip fracture. N Engl J Med 1988; 319: 1392-7.

[13] Palmer R, Saywell R, Zollinger T, et al. The impact of the prospective payment system on the treatements of hip fractures in the elderly. Arch Int Med 1990; 150: 1550-1.

[14] Shen YC. The effect of financial pressure on the quality of care in hospitals. J Health Econ 2003; 22: 243-69.

[15] Cutler D. The incidence of adverse medical outcomes under prospective payment. Econometrica 1995; 63: 29-50.

[16] Bradley E, Holmboe E, Mattera J, Roumanis S, Radford MJ, Krumholz HM. A qualitative study of increasing beta blocker use after myocardial infarction: Why do some hospitals succeed? JAMA 2001; 285: 2604-11.

[17] Needleman J, Buerhaus P, Mattke S, Stewart M, Zelevinsky K. Nurse-staffing levels and the quality of care in hospitals. N Engl J Med 2002; 346: 1715-22.

[18] Kovner C, Jones C, Zhan C, Gergen P, Baum M. Nurse staffing and postsurgical adverse events: an analysis of administrative data from a sample of U.S. hospitals, 1990-1996. Health Serv Res 2002; 37: 611-29.

[19] Aiken L, Clarke S, Cheung R, Sloane D, Silber J. Educational levels of hospital nurses and surgical patient mortality. JAMA 2003; 290: 1617-23.

[20] Person SD, Allison JJ, Kiefe CI, et al. Nurse staffing and mortality for medicare patients with acute myocardial infarction. Med Care 2004; 42: 4-12.

[21] McClellan M, Cutler D. The determinants of technological change in heart attack treatment. Working Paper 5751. Cambridge, MA: National Bureau of Economic Research 1996.

[22] Harjai KJ, Boulos L-M, Smart FW, et al. Effects of caregiver specialty on cost and clinical outcomes following hospitalization for heart failure. Am J Cardiol 1998; 82: 82-5.

[23] Auerbach AD, Hamel MB, Davis RB, et al. Resource use and survival of patients hospitalized with congestive heart failure: Differences in care by specialty of the attending physician. Ann Int Med 2000; 132: 191-200.

[24] Foody JM, Rathore SS, Wang Y, et al. Physician specialty and mortality among elderly patients hospitalized with heart failure. Am J Med 2005; 118: 1120-5.

[25] Havranek EP, Krumholz HM, Dudley RA, et al. Aligning quality and payment for heart failure care: Defining the challenges. J Cardiac Fail 2003; 9: 251-4.

[26] Hackbarth G, Reischauer R, Mutti A. Collective accountability for medical care- towards bundled medicare payments. N Engl J Med 2008; 359: 3-5. 